\title{
A Comparative Study of Eya1 and Eya4 Protein Function and Its Implication in Branchio-oto-renal Syndrome and DFNA10
}

\author{
Yuzhou Zhang, ${ }^{1}$ Boyd M. Knosp, ${ }^{2}$ Mark Maconochie, ${ }^{3}$ Rick A. Friedman, ${ }^{4}$ \\ RichaRd J.H. SMITH ${ }^{1}$ \\ ${ }^{1}$ Molecular Otolaryngology Research Laboratories, University of Iowa, Iowa City, IA 52242, USA \\ ${ }^{2}$ The University of Iowa ITS Research Service, University of Iowa, Iowa City, IA 52242, USA \\ ${ }^{3}$ School of Life Sciences, University of Sussex, Falmer, Brighton, BN1 9QG, UK \\ ${ }^{4}$ House Ear Institute, Los Angeles, CA 90057, USA
}

Received: 24 October 2003; Accepted: 29 March 2004; Online publication: 30 July 2004

\section{ABSTRACT}

Allele variants of EYA1 and EYA4, two members of the vertebrate Eya gene family, underlie two types of inherited human deafness, branchio-oto-renal (BOR) syndrome and DFNA10, respectively. To clarify how mutations in these two genes and their encoded proteins impact the normal biology of hearing, we completed a number of functional studies using the yeast-two-hybrid system. We verified that bait constructs of the homologous region (EyalHR and Eya4HR) interact with Sixl prey constructs, although no interaction with Dach1 prey was demonstrable. To compare interaction affinities, we evaluated $\alpha$-galactosidase activity after cotransformation of EyalHR/ Six 1 and Eya4HR/Six1 and found that the latter interaction was weaker. By immunofluorescence staining, we showed Eya4HR localization to the cytoplasm. After coexpression of Six1, Eya4HR was translocated to the nucleus. Results with Eya1HR were similar. Translation of mutant constructs $\left(E_{y} a 4 H R_{\mathrm{R} 564 \mathrm{X}}\right.$ and $\left.E y a 1 H R_{\mathrm{R} 539 \mathrm{X}}\right)$ could not be demonstrated. Using dual Eya-containing constructs (with two wild-type alleles or wild-type and mutant alleles), we confirmed no translation of the mutant allele, even if the mutation was nontruncating. These results are consistent with clinical data and implicate hap-

Correspondence to: Richard J.H. Smith - Department of Otolaryngology • University of Iowa • 200 Hawkins Drive • Iowa City, IA 52242. Telephone: (319) 356-3612; fax: (319) 356-4547; email: richardsmith@uiowa.edu loinsufficiency as the cause of BOR syndrome and DFNA10.

Keywords: Eya gene family, Six gene family, branchiooto-renal syndrome, DFNA10, haploinsufficiency

\section{INTRODUCTION}

The vertebrate Eya gene family is comprised of four transcriptional activators that interact with other proteins in a conserved regulatory hierarchy to ensure normal embryologic development. The structure of these proteins as deduced from their cDNA sequences includes a highly conserved 271-amino acid carboxy terminus called the eya-homologous region (eyaHR) and a more divergent proline-serine-threonine (PST)-rich $(34 \%-41 \%)$ transactivation domain at the amino terminus (eya variable region, eyaVR) (Zimmerman et al. 1997) (Fig. 1). Studies in Drosophila indicate that the eyaHR mediates interactions with the gene products of so (sine oculis) and $d a c$ (dachshund), and that expression of both eya and so is initiated by ey (eyeless) (Bonini et al. 1993, 1997; Pignoni et al. 1997). The vertebrate orthologs of so are members of the Six gene family and similarly bind with Eya proteins, inducing nuclear translocation of the resultant protein complex (Ohto et al. 1999). Amino terminal transcriptional activation also has been demonstrated for the Drosophila eya and murine 

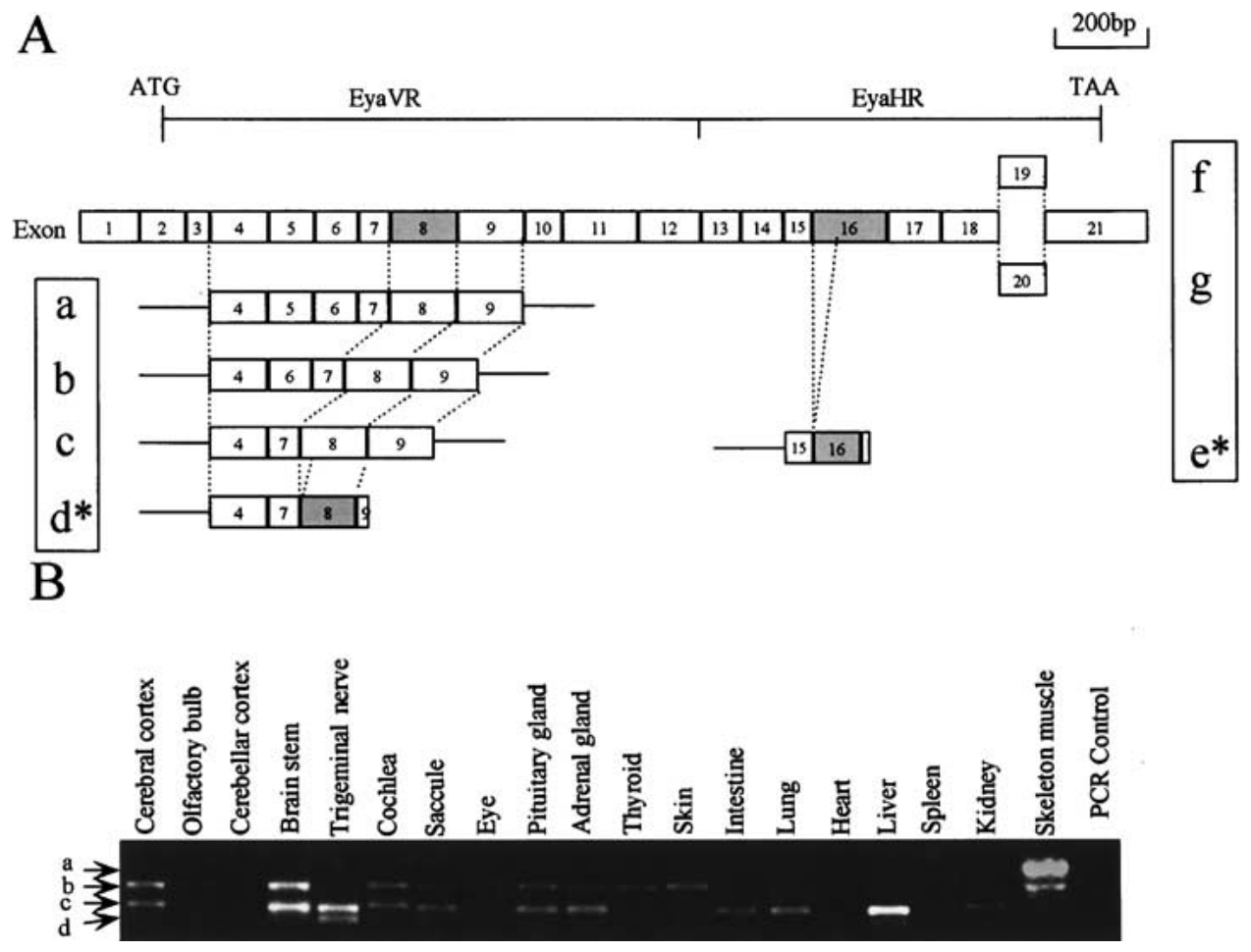

FIG. 1. Schematic illustrating the structure of the Eya4 protein showing its EyaVR and EyaHR domains. In embryonic tissue, all EyaVR isoforms $(a, b, c)$ are associated with EyaHR isoform $f$ and occasionally isoform e; in adult tissue, all EyaVR isoforms $(a, b, c)$ are associated with EyaHR isoform g. Isoform d lacks the 5' 28 base pairs of exon 8, and encodes a putative protein that lacks the EyaHR (A). Expression profiles are tissue specific (B). VR, variable region; $H R$, homologous region.
Eya1-3 gene products, an additional indication that Eya interactions and pathways are conserved across species (Pignoni et al. 1997; Xu et al. 1997).

Expression of Eya genes is present in a wide variety of tissues early in embryogenesis, and although each gene has a unique expression pattern there is extensive overlap. For example, murine studies have shown that Eya1, Eya2, and Eya4 are all expressed in the presomitic mesoderm and head mesenchyme, but only Eyal and Eya4 are expressed in the otic vesicle (Ohto et al. 1999). Eya3 expression is restricted to craniofacial and branchial arch mesenchyme in regions underlying or surrounding the Eya1-, 2-, or 4expressing cranial placodes (Heanue et al. 1999).

Allele variants of EYA1 and EYA4 underlie two types of inherited human deafness, branchio-oto-renal (BOR) syndrome (Abdelhak et al. 1997b) and DFNA10 (Wayne et al. 2001), respectively. The clinical phenotype in persons with BOR syndrome is characterized by numerous congenital anomalies involving the branchial arch system, inner and middle ears, and kidneys, with the most common features being deafness $(98.5 \%)$, preauricular pits $(83.6 \%)$, branchial anomalies (68.5\%), renal anomalies (38.2\%), and external ear abnormalities (31.5\%) (Chen et al. 1995). In persons with DFNA10, there are no abnormalities aside from late-onset hearing loss (De Leenheer et al. 2001).

These phenotypic differences are surprising given the expression profiles of EYA1 and EYA4 in embryogenesis. Both genes are expressed early in the otic vesicle in rodent inner ears, although in situ hybridization studies we have done show a spatial variability in Eya4 expression that is not seen with Eya1 expression (Wayne et al. 2001). After the otic vesicle differentiates into auditory and vestibular components, Eya 4 becomes concentrated in cells of the upper cochlear duct destined to develop into the stria vascularis and Reissner's membrane, while Eya1 is expressed in the floor of the cochlear duct in an area that gives rise to the organ of Corti. Throughout development, Eyal expression is maintained in derivatives of the neuroepithelium of the cochlear duct floor, while Eya4 expression shifts only from the upper cochlear duct to the neuroepithelium of the cochlear duct floor at embryonic day 18.5 (E18.5) (Wayne et al. 2001).

These data suggest a disjunct between the expression pattern of Eya4 and the DFNA10 phenotype. Since there is overlap in expression of Eyal and Eya 4 during embryogenesis, this disjunct may reflect functional redundancy of Eya4 to Eyal in develop- 
TABLE 1

Primers for Eya constructs

Forward

cgatcatatgtggcagtggcaagagaagt $(\mathrm{N})$

cgatcatatgtggcagtggcaagagaagt $(N)$

Caatcatatgagaagttctgggtcaaagtctcg $(\mathrm{N})$

cgatcatatgcgaggttcagatgggaagtc $(\mathrm{N})$

Caatcatatgagaagttctgggtcaaagtctcg $(\mathrm{N})$

cagtcatatgctccggatggaagaaatgat $(\mathrm{N})$

tcgatcatatggcagagatcgaaggcctaac $(\mathrm{N})$

Caatcatatgagaagttctgggtcaaagtctcg $(\mathrm{N})$

cagtcatatgctccggatggaagaaatgat $(\mathrm{N})$

Eya4HR1+Eya4HR3

Reverse

Amino acid sites

attcgtcgacacaatggatcccagctaga (S)

ctatgtcgaccggaggtggggagagatta (S)

ctatgaattcacaatggatccccagctaga (E)

tgatgaattccagtgtcaagttggcaggaa (E)

actcgaattccaaacaaaggttcgcacta (E)

actagaattcgaatcagctgggttgtcgtt $(\mathrm{E})$

ctatgaattcacaatggatccccagctaga $(\mathrm{E})$

actagaattcgaatcagctgggttgtcgtt (E)

ctatgaattcacaatggatccccagctaga $(\mathrm{E})$
$1-616$

$1-341$

$322-616$

296-591

$322-444$

384-530

493-616

$322-530$

384-616

Del 49aa (444-493)

A restriction enzyme site (underlined) was added to the $5^{\prime}$ flank of each primer. $\mathrm{N}=\mathrm{Ndel}, \mathrm{E}=\mathrm{EcoRI}, \mathrm{S}=\mathrm{Sall}$

ment but not in the mature organ system. In this article, we compare the function of Eyal and Eya 4 to clarify how mutations in these two genes and their encoded proteins impact the normal biology of hearing and result in BOR syndrome and DFNA10, respectively.

\section{MATERIALS AND METHODS}

\section{Expression profiling}

To determine whether novel isoforms of Eya4 exist, expression studies were performed against a panel of cDNA from different tissues using two sets of primers: Set 1 for detecting variants in Eya4VR (AF in exon 4,5'-TGGTTGGAGGCAGTGATACA-3'; AR in exon 9,5'-TGGCAACATCACACCAAGAT-3') and Set 2 for detecting variants in Eya4HR (BF in exon 13, 5'-

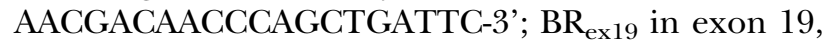
5'-GGTTAGCAGCGTGCTCCTCA-3'; and $\mathrm{BR}_{\mathrm{ex} 20}$ in exon 20, 5'-TTTTTGCTGCCTGCTCTTCT-3').

\section{Expression constructs}

To subclone Eya, total RNAwas isolated from E11 mouse embryos and skeleton muscle using the Rneasy protocol (Qiagen, Northridge, CA). Five micrograms were reverse transcribed into cDNA in a final volume of $20 \mu \mathrm{l}$ using Superscript III (Invitrogen, Carlsbad, CA) before amplifying with the following primer pairs, each designed to include restriction endonuclease sites at their 5' ends: Eya4VR, Eya4HR $R_{\text {ex } 19}$ (Eya4HR with the exon 19 splice variant), Eya $4 H R_{\text {ex20 }}$ (Eya4HR with the exon 20 splice variant), EYa4FL (full length -eyaVR and eyaHR), and Eya1HR (Table 1). For yeast-two-hybrid studies, these RT-PCR products were fused with the Gal-4 DNA-binding domain of pGBKT7 (bait). To identify the requisite portion of Eya4 for interaction with Six1, we also generated several truncated forms of Eya4HR (Table 1, Fig. 2A). Six 1 and Dach1 were rescued from Six1/RCAS and $D a c h 1 / \mathrm{pBS}$ vectors and fused with the Gal-4 activation domain of pGADT7 (prey).

To construct mammalian expression plasmids, cmyc-tagged Eya1HR or Eya4HR was subcloned into pBudCE4.1 (Invitrogen, Carlsbad, CA) under control of the CMV promoter. HA-tagged Six 1 was inserted into another multicloning site of the same vector under control of the EF- $1 \alpha$ promoter. Flag-tagged Dach1 was subcloned into p3XFIag-CMV-10 vector (Sigma-Aldrich, St. Louis, MO).

\section{Yeast-two-hybrid assays}

The MATCHMAKER 3 Gal-4 Yeast-Two-Hybrid (Y2H) system (Clontech, Palo Alto, CA) was selected for yeast interaction assays and library screening. Cotransformations were completed using the lithium acetate method to introduce each of the pGBKT7 bait constructs and each of the pGADT7 prey constructs into yeast AH109.

Yeast mating library screening was performed by single lithium acetate transformation to introduce bait Eya4HR to yeast AH109 (mat a). Transformed AH109 was selected on synthetic dropout media lacking tryptophan before mating with pretransformed yeast Y187 (mat $\alpha$ ) prey libraries derived from either mouse adult brain (Clontech, Palo Alto, CA) or mouse E10.5 otic vesicle. Mating cultures were selected on dropout media lacking tryptophan, leucine, histidine, and adenine, and containing $15 \mathrm{mM} \mathrm{3-}$ amino-triazole.

To eliminate possible false positives, yeast plasmids were extracted using a modified Qiagen miniprep protocol (Singh and Weil 2002). Potential interacting preys were rescued by electroporating to DH5 $\alpha$ competent cells on ampicillin medium. Rescued preys were reintroduced to AH109 with bait Eya4HR to 
verify the bait-prey interaction. The reporter gene assays were completed by $\alpha$-galactoside quantification as described by the manufacturer (Clontech, PaloAlto, CA).

\section{Cell cultures and immunofluorescence staining}

Human embryonic kidney (HEK) 293 cells and monkey kidney Cos-7 cells were maintained in DMEM (Gibco) supplemented with $4 \mathrm{mM} \mathrm{L}$-glutamine and $10 \%$ fetal bovine serum (Hyclone, Logan, UT). For immunofluorescence staining, cells were transfected at $30 \%-50 \%$ confluency with Fugene ${ }^{\mathrm{TM}}$ (Roche Molecular Biochemicals, Indianapolis, IN) on 4-well Chamber Slides $^{\mathrm{TM}}$ (Lab Tek, New Zealand), according to the manufacturer's instructions. After an incubation period of 24-48 h, transfected cells were fixed with $-20^{\circ} \mathrm{C}$ methanol and permeablized with $0.1 \%$ Triton X-100 in PBS. The slides were blocked in $10 \%$ BSA overnight before incubating with 1:25 diluted anti-Myc (9E10: Santa Cruz Biotechnology, Santa Cruz, CA), anti-HA (Y-11; Santa Cruz Biotechnology), or anti-FLAG (Sigma-Aldrich) antibodies, as appropriate, in PBS with 3\% BSA. Detection was achieved using a 1:200 dilution of bovine anti-mouse IgG and/or bovine anti-goat IgG conjugated with either FITC or Texas-red (Santa Cruz Biotechnology) in PBS with 3\% BSA.

\section{Mutagenesis}

Eya1 or Eya 4 mutants that have been associated with human disease were generated by site-directed mutagenesis using the Quik $^{\mathrm{TM}}$ Change protocol (Stratagene, La Jolla, CA). The forward primer and its complementary primer were used for each construct (mutagenized nucleotides are in upper case) Eya1R539X (ggaaagctgtttTgAgtgaataatccaaaggtttgg), Eya4HRR564X (gaaagttgctttgagTgaataatgcaaaggtttgg cag), Eya1HRL504R (gtaacaactacgcagcGcagcccag cattggc). Briefly, $10 \mathrm{ng}$ plasmid DNA was used for PCR cycling in a 50- $\mu \mathrm{l}$ volume with pfu Turbo DNA polymerase containing $125 \mathrm{ng}$ of each primer and $0.2 \mathrm{mM}$ dNTPs. The mutated plasmid template was digested with Dpnl $(10 \mathrm{U})$ to remove the parental strain and used to transform XL1-Blue competent cells. All mutant constructs were confirmed by sequencing.

\section{Western blotting}

Yeast lysates from transformed strain AH109 were extracted using glass beads in extraction buffer (200 $\mathrm{mM}$ Tris $\mathrm{pH} 8.0,150 \mathrm{mM}$ ammonium sulfate, $10 \%$ glycerol, 2 mM DTT, 1 mM EDTA) supplemented with protease inhibitor cocktail for fungi (Sigma-Al- drich). Whole-cell protein lysates from transfected HEK 293 cells were extracted using RIPA buffer $(1 \times$ PBS, 1\% Nonidet P-40, 0.5\% sodium deoxycholate, $0.1 \%$ SDS) supplemented with a cocktail of protease inhibitors (Complete; Roche Molecular Biochemicals). All lysates were quantified using the Bradford method (Bio-Rad, Hercules, CA).

Forty micrograms of total protein were resolved on 10\% SDS-PAGE gel and transferred to a nitrocellulose membrane (Protran 0.2- $\mu \mathrm{m}$ pore size; Schleicher \& Schuell, Keene, NH). The membranes were blocked with $5 \%$ milk in TBST $(50 \mathrm{mM}$ Tris- $\mathrm{HCl} \mathrm{pH}$ $7.4,150 \mathrm{mM} \mathrm{NaCl}, 0.5 \%$ Tween-20) at $4^{\circ} \mathrm{C}$ overnight. Blots were incubated with primary antibody (1:500; Santa Cruz Biotechnology) for $1 \mathrm{~h}$ at $25^{\circ} \mathrm{C}$ and then washed three times with TBST. Secondary antibody conjugated with HRP (1:5000; Jackson Immunoresearch, West Grove, PA) was incubated for $45 \mathrm{~min}$ at $25^{\circ} \mathrm{C}$. Visualization was carried out with enhanced chemiluminescence (ECL, Amersham Pharmacia Biotech, Piscataway, NJ).

\section{Protein modeling}

The hypothetical structures for EYAl and its mutations were created using Swiss-Pdb Viewer (GlaxoSmithKline, www.expasy.org/spdbv). Structure optimization and modifications were made using Sybyl software (Tripos Associates, St. Louis, MO).

\section{RESULTS}

\section{Novel Eya4 isoforms}

We identified several tissue-specific Eya4 isoforms by RT-PCR and verified results by sequencing of multiple clones. Although many of these splice variants have been reported previously (Borsani et al. 1999), two novel isoforms were seen isoform c-f and c-g, splice variants that exclude exon 6 (the inclusion or exclusion of exon 6 maintains the same open reading frame), and isoform d, a splice variant deleting the 5 ' 28 base pairs of exon 8 . The putative protein generated by isoform d lacks the Eya homologous region and was cloned from an E11 murine embryonic cDNA library.

We demonstrated strong murine skeletal muscle expression of isoform a-g, which other investigators have reported as absent (Borsani et al. 1999). We also confirmed our earlier studies showing alternative splicing of exon 19 and exon 20 (isoforms $f$ and $g$ ) in different tissues, with expression of isoform $f$ in embryonic cells and isoform $\mathrm{g}$ in mature cells (Wayne et al. 2001). Isoforms b-f and c-f are expressed in fetal cochlea and b-g and c-g in adult cochlea (Fig. 1). 
A

\begin{tabular}{|c|c|c|c|}
\hline EyaVR & EyaHR & Autoactivation & Six1 interaction \\
\hline Eya4FL & & +++ & $\mathrm{n} / \mathrm{a}$ \\
\hline Eya4VR & & +++ & $\mathrm{n} / \mathrm{a}$ \\
\hline Eya4HR & & - & +++ \\
\hline EyalHR & & - & +++ \\
\hline Eya4HR1 & & - & - \\
\hline Eya4HR2 & & - & - \\
\hline Eya4HR3 & & - & - \\
\hline Eya4HR4 [Eya4HR $(\mathrm{R} 564 \mathrm{X})]$ & & - & - \\
\hline Eya4HR5 & & - & - \\
\hline Eya4HR6 & & - & - \\
\hline
\end{tabular}

B

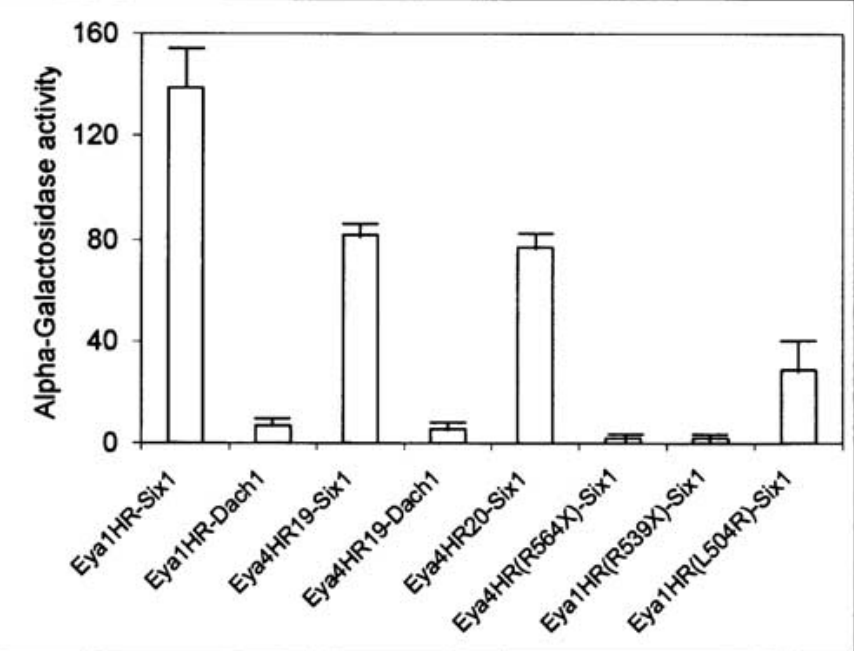

C

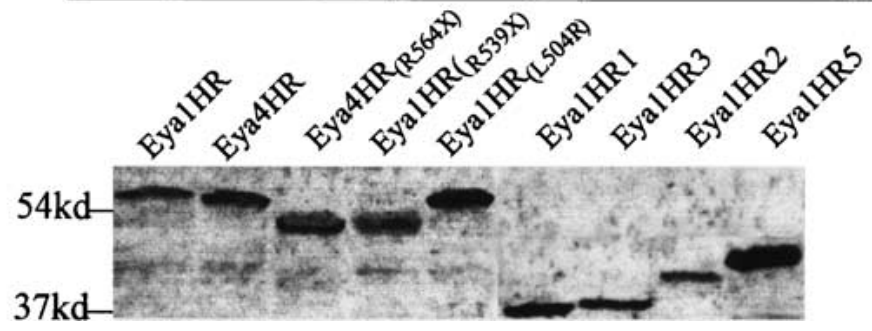

FIG. 2. Schematic of the $\mathrm{Y} 2 \mathrm{H}$ bait constructs of Eya4. Constructs containing EyaVR autoactivated and were unsuitable as baits. None of the partial EyaHR constructs (Eya4HR1-6) generated proteins that interacted with Six1 (A). Interaction strengths were measured by assaying $\alpha$-galactosidase activity after cotransformation of Eya (wildtype or mutant baits) with Six1 (prey) or Dach1 (prey). Nonsense

\section{Interaction between Eya1/Eya4 and Six1}

We designed bait constructs of Eya4 that included the entire coding region, only the eyaVR, and only the eyaHR. Although all constructs that contained the eyaVR autoactivated, Eya4 constructs that contained only the eyaHR showed slight "leakage" of the His report gene that could be suppressed by adding $15 \mathrm{mM}$ 3-AT (Fig. 2A). The Eyal constructs we designed showed a similar autoactivation profile. mutant constructs of Eya did not interact with Six1, and with the L504R missense mutation of Eya1, interaction was reduced by 75 of expected levels. Neither Eya1HR nor Eya4HR baits interacted with Dach 1 prey $(\mathbf{B})$. Immunoblotting of yeast extracts verified expression of wild-type and mutant proteins $(\mathbf{C})$. FL, full length; VR, variable region; $\mathrm{HR}$, homologous region.

To identify Eya4 interacting partners, we used the Eya4HR construct as bait against an otic vesicle prey library (pGADT7; E10.5) in a Y2H mating screen. Mating efficiency was $2.5 \%$ and total screenable diploids were $1.6 \times 10^{7}$. One hundred sixty-five Ade/His/ MEL1 positive preys were rescued and subjected to second-round screening to eliminate (a) autoactivating prey by single transformation and (b) false positives by reintroducing one-by-one prey and Eya4HR bait. After second-round screening, three positive clones were identified that encoded the Six1 protein, 


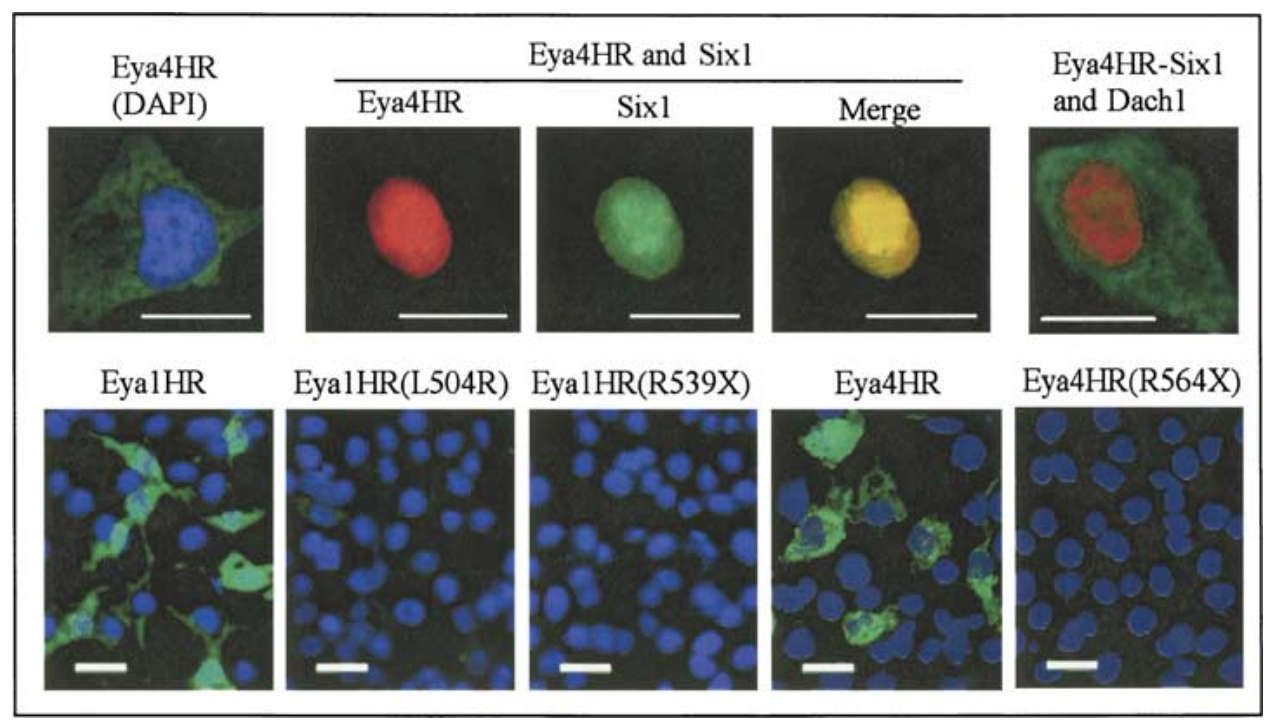

FIG. 3. Subcellular localization and translocation of wild-type and mutant Eya proteins in Cos-7 cells. Top row: Single transfection of cmyc-tagged Eya4HR shows cytoplasmic localization of the translated protein (green; blue, DAPI staining of the nucleus). Cotransfection of C-myc-tagged Eya4HR with HA-tagged Six 1 results in nuclear translocation of the Eya4HR/Six1 protein complex (EyaHR, red; Six1, green), as the merged image (yellow) shows. Coexpression of

but other Six or Dach homologs were not identified. We verified the presence of Dach1 in the otic vesicle prey library by PCR, although its expression was weaker than Sixl (data not shown).

We evaluated $\alpha$-galactosidase activities of the MEL 1 reporter gene after cotransformation of yeast AH109 with Eya1HR/Six 1 and Eya4HR/Six 1 and documented in vivo interaction of both EyalHR and Eya4HR proteins with Six1. Of the two interactions, Eya1HR/ Six1 was stronger (Fig. 2B). The interaction of the different Eya4 isoforms (Eya $4 H R_{\mathrm{Ex} 19}$ and $\left.E y a 4 H R_{\mathrm{Ex} 20}\right)$ with Six1 was comparable. We tested but could not demonstrate a direct interaction of Eya1HR or Eya4HR protein with Dach1 using our Y2H system (Fig. 2B).

\section{EyaHR and function}

To determine the impact of the DFNA10-causing human EYA4 (R587X) mutation, we generated an EYA4HR ${ }_{\text {R564X }}$ construct with mutation 2200CGA $>\rightarrow$ TGA (mouse Eya4 (R564X) [NP_034297] corresponding to human EYA4[NP_004091]). This construct generates a truncated protein missing the 53 carboxy-terminal amino acids of Eya4. Using our $\mathrm{Y} 2 \mathrm{H}$ system, we were unable to demonstrate an interaction between Eya4HR $\mathrm{R}_{564 x}$ protein and Six1 (Fig. 2B). To investigate whether this finding was Eya4 specific, we generated a mutant bait construct of EyalHR that contained the corresponding amino acid change (mouse Eya1 (R539X) [NP_034294]). This truncated
Eya4HR, Six1, and Dach1 shows flag-tagged Dach1 (green) is not recruited with the Eya4HR/Six 1 protein complex into the nucleus (Eya4HR, red) (scale bars, $10 \mu \mathrm{m}$ ). Bottom row: Wild-type Eya1HR and Eya4HR proteins localize to the cytoplasm. Translation of the Eya1HR $R_{\mathrm{L} 504 \mathrm{R}}$ bait construct is markedly reduced and translation of the Eya1HR $R_{\text {R39x }}$ and Eya4HR $R_{\text {R644X }}$ bait constructs is essentially nonexistent.

protein also did not interact with Six1. Expression of both mutant Eya proteins was demonstrable in our Y2H system (Fig. 2C).

To determine whether a BOR-causing missense mutation abrogates the Six1 interaction, we next generated an Eya1HR $R_{\mathrm{L} 504 \mathrm{R}}$ bait construct (mouse Eyal (L504R) [NP_034294] corresponding to human (EYA1 (L472) [NP_742057]) as this mutation causes BOR syndrome with typical phenotypic features (Argiriadi et al. 1999). Using the Y2H system, we found that although the interaction between Eya1HR $_{\text {L504R }}$ protein and Six1 was not totally abolished, it was significantly reduced (Fig. 2B). As truncated EyaHR (loss of a small portion of the carboxy-terminus) did not interact with Six1, we sought to determine the requisite portion of the EyaHR protein for Six1 interaction by generating and testing several Eya4HR truncated constructs (EyaHR1-6; Fig. 2A). None was found to interact with Six1 (data not shown), although these mutant proteins were expressed (Fig. 2C).

\section{Colocalization studies}

We studied Eya localization in mammalian cells by immunofluorescence staining. Forty-eight hours after transfection, c-myc-tagged Eya4HR protein could be demonstrated in the cytoplasm, and after coexpression of HA-tagged Six1, it was translocated to the nucleus (Fig. 3). Flag-tagged Dach1 was localized in the cytoplasm and, when expressed with Six1 and 


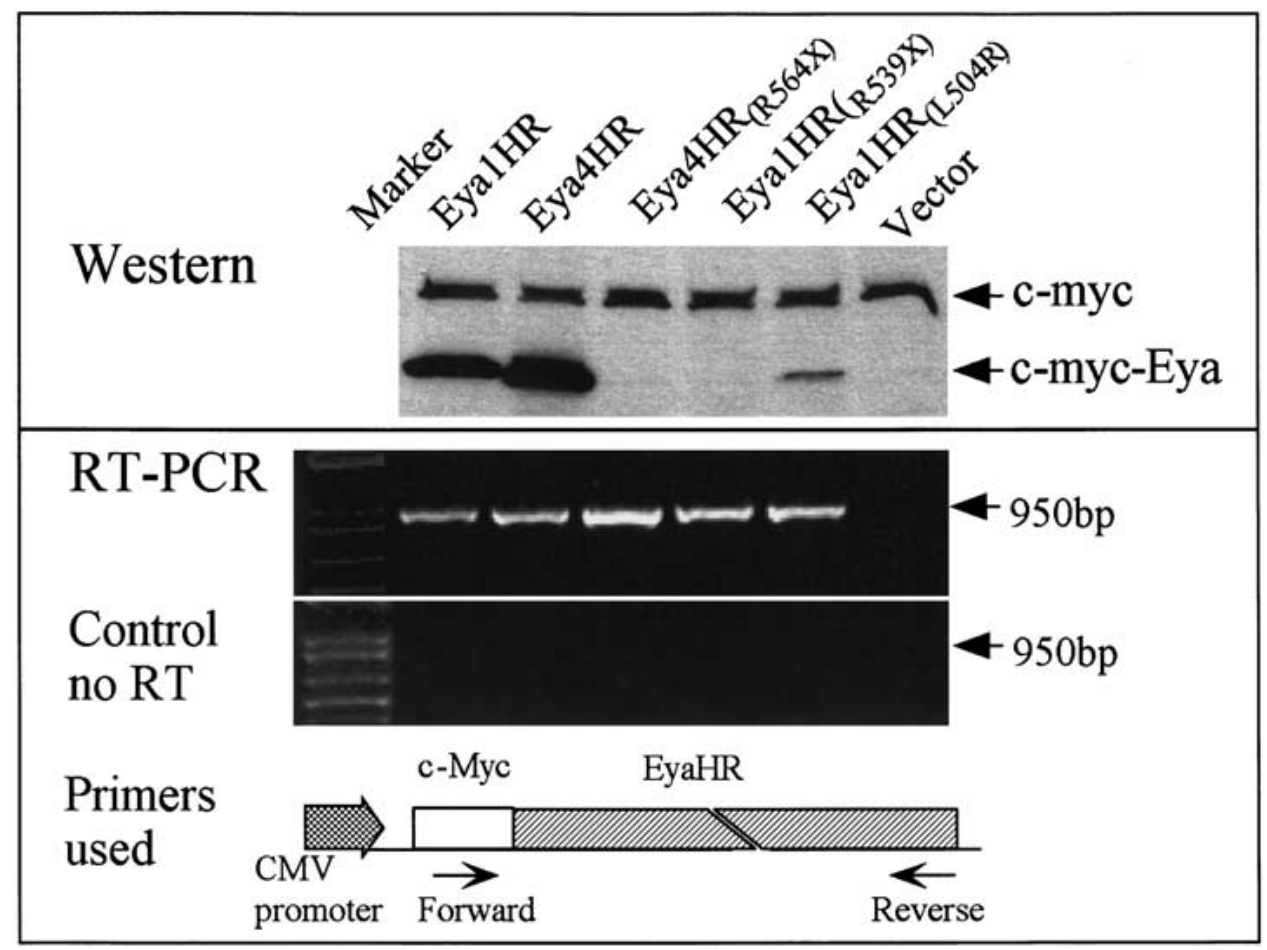

FIG. 4. Top row: Rapid degradation of mutant Eya protein after translation is apparent by immunoblotting $4 \mathrm{~h}$ after transfection (detection-primary antibody: mouse anti-c-myc antibody; secondary antibody: HRP-conjugated goat anti-mouse antibody). Bottom row: A parallel RT-PCR assay on total RNA extracted from HEK293

Eya4HR, did not colocalize with the Eya4HR/Six1 protein complex (Fig. 3). When we tested c-myctagged Eya1HR, we confirmed reports demonstrating localization of Eya1HR protein to the cytoplasm with nuclear translocation when coexpressed with Six1 protein (data not shown) (Buller et al. 2001).

${\text { Eya } 4 H R_{\text {R564X }} \text { and Eya1HR }}_{\text {R539x proteins could not }}$ be demonstrated in transfected HEK 293 cells (Fig. 3). These results were confirmed with several independent clones and transfections and replicated using several other truncated constructs of Eya $4 H R$. We also noted only very low fluorescence intensity of the translated protein using the BOR-causing EyalHR $_{\mathrm{L} 504 \mathrm{R}}$ missense construct as compared to normal constructs (Fig. 3).

\section{Mutant protein degradation}

Translation of EyaHR mutant constructs containing nonsense mutations could not be demonstrated in transfected cells by immunoblot analysis. While we could detect wild-type c-myc-tagged Eya1HR and Eya4HR proteins as early as $4 \mathrm{~h}$ post-transfection, ${\text { Eya } 4 \mathrm{HR}_{\mathrm{R} 564 \mathrm{X}} \text { and Eya1HR }}_{\mathrm{R} 539 \mathrm{x}}$ proteins could not be detected at 4 or $16 \mathrm{~h}$ post-transfection. Eya1HR $\mathrm{L}_{504 \mathrm{R}}$ protein was detectable as a very weak band (Fig. 4). cells transfected with Eya constructs (wild-type and mutant) shows robust amplification of both wild-type and mutant tagged Eya1HR and Eya4HR mRNA using primers specific for the expressed fusion protein, while in the control (no RT), no amplification is seen.

Levels of tagged mRNA from wild-type constructs and mutant constructs showed comparable expression intensities using primers specific for the fusion proteins (Fig. 4).

Haploinsufficiency causes the BOR and DFNA10 phenotypes

As mutations in EyaHR appeared to be associated with rapid protein degradation, we sought to determine whether an EyaHR truncated mutant has a dominant-negative effect on a wild-type allele. We designed two dual Eya constructs, one containing two wild-type EyaHR (one with a c-myc tag and one with an HA tag) and one containing wild-type c-myctagged EyaHR and HA-tagged EyaHR R564X. Transfection efficiency with both dual constructs was identical but in the mutant-containing construct only the c-myc-tagged protein was identified. To verify this result, we used Western blotting to semiquantitate c-myc-tagged and HA-tagged Eya4HR proteins and could not demonstrate mutant HAtagged Eya4HR (Fig. 5). These findings exclude a dominant-negative effect as the cause of BOR syndrome or DFNA10. 


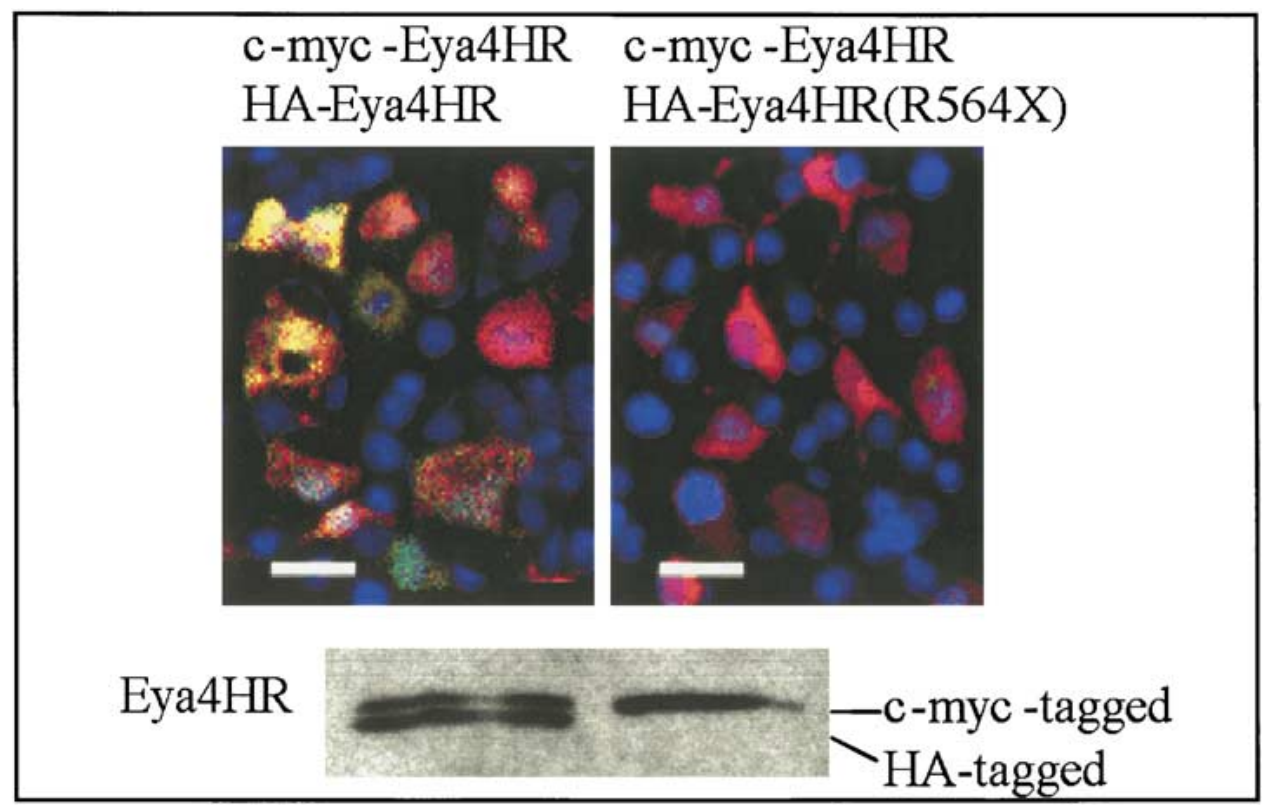

FIG. 5. Top row: pBudCE4.1 containing two wild-type EyaHR inserts (one with a c-myc tag and one with an HA tag) show good translation of both proteins (yellow) in most cells. When the HAtagged wild-type EyaHR insert is replaced with HA-tagged Eya4HR $R_{\mathrm{R} 564 X}$, although transfection efficiency remains similar, the

\section{Molecular modeling}

Hypothetical models for Eya1, Eya1 $1_{\mathrm{L} 504 \mathrm{R}}$, and Eya $1_{\text {R539X }}$ proteins were created using Swiss-PdbViewer (GlaxoSmithKline, www.expasy.org/spdbv) to thread the amino acid sequence of Eyal onto the known structure of epoxide hydrolase (www.pdb.org PDB ID: 1CQZ), which was a match for Eyal in the NCBI conserved domain database (www.ncbi.nlm. nih.gov/Structure/cdd/cdd.shtml) (Abdelhak et al. 1997a). Compared with the Eyal $1_{\mathrm{R} 539 x}$ protein, the model of Eyal $1_{\mathrm{L} 504 \mathrm{R}}$ shows a change secondary to the point mutation that moves the carboxy-terminal section truncated in Eyal ${ }_{\text {R539x }}$ to the other side of the molecule, thereby causing similar alterations in structure (Fig. 6).

\section{DISCUSSION}

The mechanism by which a mutation produces a dominant phenotype is seldom intuitively clear, but as a general rule these types of mutations cause either loss or gain of function. Loss of function typically implies haploinsufficiency through reduced gene dosage, expression, or protein activity, while gain of function implies increased gene dosage, altered mRNA expression, increased or abnormal protein activity, or dominant-negative effects (Wilkie 1994). Particularly vulnerable to haploinsufficiency are regulatory genes working close to threshold levels in
HA-tagged Eya4HR $\mathrm{R}_{564 x}$ protein cannot be identified (scale bars, $20 \mu \mathrm{m})$. Bottom row: Cell extracts subjected to immunoblotting with two primary monoclonal antibodies against c-myc tag (9E10) and HA-tag (F-7) show no translation of the mutant construct (right).

specific tissues. Examples include $P A X 3$, which is responsible for Waardenburg syndrome Type 1 (Tassabehji et al. 1995), and SOX9, a cause of campomelic dysplasia (Sock et al. 2001). Based on our data, EYA1 and EYA4 can be added to this list.

Using the $\mathrm{Y} 2 \mathrm{H}$ system, we confirmed the reported interaction of Eya1HR/Six1 (Buller et al. 2001) and showed that Six1 protein also interacts, albeit more weakly, with Eya4HR protein. We identified the Eya4HR/Six1 protein interaction by screening an E10.5 otic vesicle prey library and found that both Eya4 isoforms (Eya4HR $R_{\mathrm{Ex} 19}$ and $\left.E y a 4 H R_{\mathrm{Ex} 20}\right)$ have comparable affinity for Six1, although only the exon 19-containing variant is present in the developing inner ear (Wayne et al. 2001). This functional redundancy of Eya4 to Eyal with respect to Sixl is consistent with the absence of a congenital phenotype in persons with DFNA10 deafness (De Leenheer et al. 2001).

Other investigators have proposed an EyalHR/ Dach1 interaction (Chen et al. 1997), which we could not verify in our $\mathrm{Y} 2 \mathrm{H}$ system. We were also unable to demonstrate direct binding of Eya4HR and Dach1 proteins and found that Dach1 did not colocalize with the Eya4HR/Six1 protein complex. Interestingly, an Eya1/Dach1 interaction has been demonstrated using a mammalian 2-hybrid system (Ozaki et al. 2002), suggesting that either the full-length protein or EyaVR is required to interact with Dach1, or that the interaction between Dach1 and EyaHR re- 


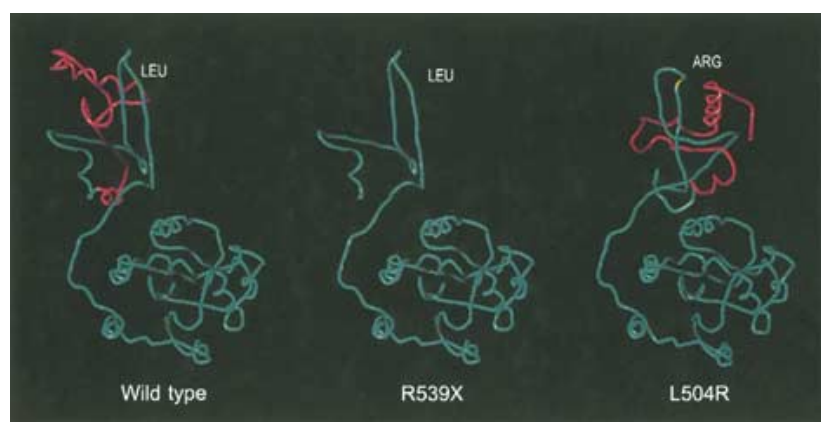

FIG. 6. The likely structures (backbone only) for Eya1, Eya $1_{\mathrm{R} 539 \mathrm{X}}$ and Eya $1_{\mathrm{L} 504 \mathrm{R}}$ proteins are shown. In purple is the section of the protein that is truncated in Eya $1_{\mathrm{R} 539 \mathrm{X}}$, in green is the site of the point mutation, which is depicted as yellow in Eya1 $1_{\text {L504R. The hypothetical }}$ change due to the point mutation moves the section truncated in Eya $1_{\mathrm{R} 539 \mathrm{X}}$ to the other side of the molecule in Eya $1_{\mathrm{L} 504 \mathrm{R}}$.

TABLE 2

Correlation between BOR syndrome phenotypes and genotypes

\begin{tabular}{lcc}
\hline & \multicolumn{2}{c}{ Phenotype } \\
\cline { 2 - 3 } Genotype & Severe & Mild \\
\hline Truncating mutations & 19 & 6 \\
Nontruncating mutations & 8 & 2 \\
\hline
\end{tabular}

$p=0.799$.

quires a third protein that is not expressed in the experimental systems we used.

By generating truncating mutant constructs $\left(E_{y a} 4 H R_{\mathrm{R} 564 \mathrm{x}}\right.$ and $\left.E y a 1 H R_{\mathrm{R} 539 \mathrm{X}}\right)$, we abolished the interaction of Eya proteins with Six1. Based on this finding, we hypothesized that a Six1 domain could be defined, but although we made numerous partial constructs of the EyaHR domain that were expressed, we were unable to identify a Six1 binding site. Based on these data, we believe the Eya/Six protein-protein interaction is dependent on the juxtaposition of several distant amino acids of Eya brought in proximity by the three-dimensional conformation of the homologous region. In support of this hypothesis, we found that the missense construct Eya1HR $R_{\mathrm{L} 504 \mathrm{R}}$ also had significantly reduced Six1 affinity, presumably on the basis of a conformational change (Fig. 6).

Translation of the two EyaHR nonsense-containing mutant constructs could not be demonstrated in transfected cells by immunoblot analysis and the missense Eya1HR $\mathrm{L}_{\mathrm{L} 54 \mathrm{R}}$ protein was detectable only as a very weak band (Fig. 4). Because levels of tagged mRNA from wild-type and mutant constructs showed comparable expression intensities using a pair of tagged gene-specific primers (Fig. 4), we believe that mutant proteins are rapidly degraded and that haploinsufficiency causes the BOR and DFNA10 phenotypes. Additional in vitro support for this hypothesis comes from studies using dual EyaHR constructs. While we could demonstrate equivalent transfection efficiency, we could not identify the HA-tagged Eya1HR $_{\mathrm{L} 504 \mathrm{R}}$ mutant protein either by immunostaining or by Western blotting (Fig. 5).

The BOR-typical L504R mutation represents a decrease in hydrophobicity and an increase in electrostatic charge and size of the amino acid side chain at position 504. These changes affect the interaction of the mutation site with neighboring amino acids and the surrounding environment, and on this basis the resultant three-dimensional protein structure of Eya $_{\text {L504R }}$ appears significantly altered. Major differences in protein conformation are also predicted by trypsin digestion studies that show a low sensitivity to but distinct pattern of digestion of the Eyal $1_{\text {L504R }}$ mutant protein compared with wild-type protein (Ozaki et al. 2002). Similarities in the protein structure of Eyal $1_{\mathrm{R} 539 \mathrm{X}}$ and $\mathrm{Eya}_{\mathrm{L} 504 \mathrm{R}}$ are consistent with the rapid degradation we observed (Fig. 6).

Haploinsufficiency as the cause of BOR syndrome is supported by genotype-phenotype studies based on the different allele variants of EYA1 in persons segregating a BOR phenotype. Notably included in this list of 51 different mutations are 23 frameshift or nonsense mutations, 8 missense mutations, 9 splice site mutations, and 11 complex mutations involving large deletions or chromosomal rearrangements (www.medicine uiowa.edu/pendredandbor). Although Abdelhak et al. (1997a) hypothesized that the majority of BORcausing mutations cluster in the eyaHR, they are in fact randomly scattered throughout the gene, with complex rearrangements involving every exon and point mutations and small insertions/deletions occurring in every exon except for exons 1-3.

The clinical phenotype that results from these mutations variably affects the branchial arch system, inner and middle ears, and kidneys. If we define classical BOR syndrome as the presence of at least three of these cardinal features (Chen et al. 1995) and define subgroup genotypes as inactivating or noninactivating (inactivating-splice site mutations, insertions, nonsense mutations, and duplications and deletions of more than 3 bp; noninactivating mutations-missense mutations and 3-bp deletions), we can compare phenotype with genotype to show that inactivating mutations are not associated with a more severe clinical disease spectrum $(p=0.799$; Table 2$)$. A similar comparative study with DFNA10-causing EYA4 mutations is not possible as only three families have been reported to segregate deafness at this locus. The responsible mutations include a nonsense mutation and two frame shift-causing deletions, all of 
which affect the eyaHR (Wayne et al. 2001; Pfister et al. 2002).

In conclusion, our data suggest that the functional integrity of EyaHR depends on its three-dimensional conformation. Although autoactivation of Eya $4 F L$ and EyaVR constructs precluded their use as bait in our Y2H system, we studied multiple EyaHR constructs. Both Eya1HR and Eya4HR proteins bind Six1, with subsequent nuclear translocation. Six 1, in turn, can bind to specific DNA promoters, with an affinity that may be modulated by EyaVR. We observed no EyaHRSix1 interaction with Dach1. Furthermore, in our Y2H system the EyaHR mutant proteins we studied did not interact with Six1, and in the mammalian system were rapidly degraded. In concurrence with clinical data, our findings imply that haploinsufficiency is the basis for the BOR and DFNA10 phenotypes.

\section{ACKNOWLEDGMENTS}

This research was supported in part by NIH grant R01DC03544 (RJHS).

\section{REFERENCES}

Abdelhak S, Kalatzis V, Heilig R, Compain S, Samson D, Vincent C, Levi-Acobas F, Cruaud C, Le Merrer M, Mathieu M, Konig R, Vigneron J, Weissenbach J, Petit C, Weil D. Clustering of mutations responsible for Branchio-Oto-Renal (BOR) syndrome in the eyes absent homologous region (eyaHR) of EYA1. Hum. Mot. Genet. 6:2247-2255, 1997a.

abdelhak S, Kalatzis V, Heilig R, Compain S, Samson D, Vincent C, Weil D, Cruaud C, Sahly I, Leibovici M, Bitner-Glindzicz M, Francis M, Lacombe D, Vigneron J, Charachon R, Boven K, Bedbeder P, Van Regemorter N, Weissenbach J, Petit C. A human homologue of the Drosphila eyes absent gene underlies branchio-oto-renal (BOR) syndrome and identifies a novel gene family. Nat. Genet. 15:157-164, 1997b.

Argiriadi MA, Morisseau C, Hammock BD, Christianson DW. Detoxification of environmental mutagens and carcinogens: structure-based mechanism and evolution of liver epoxide hydrolase. Proc. Natl. Acad. Sci. USA 96:10637-10642, 1999.

Bonini N, Leiserson W, BenXer S. The eyes absent gene: genetic control of cell survival and differentiation in the developing Drosophila eye. Cell 72:971-982, 1993.

Bonini NM, Quang T, GraY-Board GL, Warrick JM. The Drosophila eyes absent gene directs ectopic eye formation in a pathway conserved between flies and vertebrates. Development 124:4819-4826, 1997.

Borsani G, Degrani A, Ballabio A, Bulfone A, Bernard L, Banfi S, Gattuso C, Mariani M, Dixon M, Donnai D, Metcalfe K, Winter R, Robertson M, Axton R, Brown A, Van Heyningen V, Hanson I. Eya4, a novel vertebrate gene related to Drosophila eyes absent. Hum. Mol. Genet. 8:11-23, 1999.
Buller C, Xu X, Marquis V, Schwanke R, Xu P-X. Molecular effects of Eyal domain mutations causing organ defects in BOR syndrome. Hum. Mol. Genet. 10:2775-2781, 2001.

Chen AH, Francis M, Ni L, Cremers CWRJ, Kimberling WJ, Sato Y, Phelps PD, Bellman SC, Wagner MJ, Pembrey M, Smith RJH. Phenotypic manifestations of branchiootorenal syndrome. Am. J. Med. Genet. 58:365-370, 1995.

Chen R, Amoui M, Zhang Z, Mardon G. Dachshund and eyes absent proteins form a complex and function synergistically to induce ectopic eye development in Drosophila. Cell 91:893-903, 1997.

De Leenheer EMr, Huygen PlM, Wayne S, Smith RJH, Cremers CWRJ. The DFNA10 phenotype. Ann. Otol. Rhinol. Laryngol. 110:861-866, 2001.

Heanue TA, Reshef R, Davis RJ, Mardon G, Oliver G, Tomarev S, LASSAR AB, TABIN CJ. Synergistic regulation of vertebrate muscle development by Dach2, Eya2, and Six1, homologs of genes required for Drosphila eye formation. Genes Dev. 13:3231-3243, 1999.

Ohto H, Kamada S, Tago K, Tominaga SI, Ozaki H, Sato S, Kawakami $\mathrm{K}$. Cooperation of Six and Eya in activation of their target genes through nuclear translocation of Eya. Mol. Cell. Biol. 19:6815$6824,1999$.

Ozaki H, Watanabe Y, Ikeda K, Kawakami K. Impaired interactions between mouse Eyal harboring mutation found in patients with branchio-oto-renal syndrome and Six, Dach, and G proteins. J. Hum. Genet. 47:107-116, 2002.

Pfister M, Toth T, Thiele H, HaAck B, Blin N, Zenner HP, Sziklai I, Mumberg P, Kupka S. A 4-bp insertion in the eyahomologous region (eyaHR) of EYA4 causes hearing impairment in a Hungarian family linked to DFNA10. Mol. Med. 8:607-611, 2002.

Pignoni F, Hu B, Zavitz KH, Xiao J, Garrity PA, Zipursky SL. The eye-specification proteins So and Eya from a complex and regulate multiple steps in Drosophila eye development. Cell 91:881891, 1997.

SINGH MV, WeIL AP. A method for plasmid purification directly from yeast. Anal. Biochem. 307:13-17, 2002.

Sock E, Pagon RA, Keymolen K, Lissens W, Wegner M, Scherer G. Loss of DNA-dependent dimerization of the transcription factor SOX9 as a cause for campomelic dysplasia. Hum. Mol. Genet. 12:1439-1447, 2003.

Tassabehji M, Newton VE, Liu XZ, Brady A, Donnai D, KrajewskaWalasek M, Murday V, Norman A, Obersztyn E, Reardon W. The mutational spectrum in Waardenburg syndrome. Hum. Mol. Genet. 4:2131-2137, 1995.

Wayne S, Robertson NG, Declau F, Chen N, Verhoeven K, Prasad S, Tranebjarg L, Morton C, Ryan AF, Van Camp G, Smith RJH. Mutations in the transcriptional activator EYA4 cause late-onset deafness at the DFNA10 locus. Hum. Mol. Genet. 10:195-200, 2001.

WILKIE AOM. The molecular basis of genetic dominance. J. Med. Genet. 31:89-98, 1994.

Xu P-X, Woo I, Her H, Beier D, Maas R. Mouse Eya homologues of the Drosophila eyes absent gene require Pax6 for expression in cranial placodes. Development 124:219-231, 1997.

Zimmerman Je, Bui QT, Steingrimsson E, Nagle DL, Fu W, Genin A, Spinner NB, Copeland NG, Jenkins NA, Bucan M, Bonini NM. Cloning and characterization of two vertebrate homologs of the Drosophila eyes absent gene. Gen. Res. 7:128141, 1997. 